\title{
NLRP3 Inflammasome as a Common Denominator of Atherosclerosis and Abdominal Aortic Aneurysm
}

\author{
Masafumi Takahashi, MD, PhD
}

\begin{abstract}
Atherosclerosis and abdominal aortic aneurysm (AAA) are multifactorial diseases characterized by inflammatory cell infiltration, matrix degradation, and thrombosis in the arterial wall. Although there are some differences between atherosclerosis and AAA, inflammation is a prominent common feature of these disorders. The nucleotide-binding oligomerization domain-like receptor family pyrin domain containing 3 (NLRP3) inflammasome is a cytosolic multiprotein complex that activates caspase-1 and regulates the release of proinflammatory cytokines interleukin (IL)- $1 \beta$ and IL-18, as well as the induction of lytic cell death, termed pyroptosis, thereby leading to inflammation. Previous experimental and clinical studies have demonstrated that inflammation in atherosclerosis and AAA is mediated primarily through the NLRP3 inflammasome. Furthermore, recent results of the Canakinumab Anti-inflammatory Thrombosis and Outcome Study (CANTOS) showed that IL-1 $\beta$ inhibition reduces systemic inflammation and prevents atherothrombotic events; this supports the concept that the NLRP3 inflammasome is a promising therapeutic target for cardiovascular diseases, including atherosclerosis and AAA. This review summarizes current knowledge with a focus on the role of the NLRP3 inflammasome in atherosclerosis and AAA, and discusses the prospects of NLRP3 inflammasome-targeted therapy.
\end{abstract}

Key Words: Cytokines; Inflammation; Interleukins; Pyroptosis; Vasculature

C ardiovascular disease (CVD) is the leading cause of death globally, and it has been estimated that half CVD, including myocardial infarction (MI), heart failure, stroke, and peripheral arterial diseases (PAD), can be attributed to atherosclerosis, which is characterized by endothelial dysfunction, lipid accumulation, inflammatory cell infiltration, and proliferation of vascular smooth muscle cells (VSMCs) in the arterial wall. Although dyslipidemia is a major cause of atherosclerosis, accumulating evidence indicates that inflammation is also a main driver of atherosclerosis; therefore, it is generally accepted that atherosclerosis is a chronic inflammatory disease in the arterial wall. ${ }^{1}$ Conversely, abdominal aortic aneurysm (AAA) is characterized by progressive dilatation of the aortic wall, and remains a significant cause of death in the elderly. Because atherosclerotic changes and inflammatory cell infiltration are commonly seen in the arterial wall of AAA patients, AAA is also regarded as a chronic inflammatory disease. ${ }^{2,3}$ Furthermore, patients with AAA often have atherosclerosis, such as coronary artery disease and PAD, suggesting a possible link in the pathogenesis of atherosclerosis and AAA. However, it is still unclear whether atherosclerosis is a causal factor of AAA or simply that a similar mechanism underlies atherosclerosis and AAA. ${ }^{4,5}$ Although there are some differences between atherosclerosis and AAA, inflammation is a prominent common feature. In particular, because inflammation in atherosclerosis and AAA usually occurs even in the absence of microbial infection, this type of inflammation has been referred to as sterile inflammation.

Emerging evidence indicates that the nucleotide-binding oligomerization domain-like receptor family pyrin domain containing 3 (NLRP3) inflammasome is a key driver of sterile inflammation in a wide variety of diseases, including autoinflammatory disease, CVD, metabolic and kidney diseases, and neurological disorders. ${ }^{68}$ The NLRP3 inflammasome is a cytosolic molecular complex that is formed in response to various danger signals, known as pathogen-associated molecular patterns (PAMPs) or damage/danger-associated molecular patterns (DAMPs). Once the NLRP3 inflammasome is formed and activated, it activates the cysteine protease caspase- 1 , which converts prointerleukin (IL)- $1 \beta$ to its active form. Because IL- $1 \beta$ is a potent proinflammatory cytokine, the release of IL- $1 \beta$ leads to inflammation in the surrounding tissues. Previous investigations from our and other groups have demonstrated that the NLRP3 inflammasome and the downstream cytokine IL- $1 \beta$ play a pivotal role in the pathogenesis of CVD, including atherosclerosis and AAA;9-12 this suggests that NLRP3 inflammasome-driven IL- $1 \beta$ is a potential therapeutic target for CVD. Indeed, recent results of the Canakinumab Anti-inflammatory Thrombosis and Outcome Study (CANTOS) have proven that IL-1 $\beta$ inhibition reduces the incidence of atherothrombotic events in prior MI patients with residual inflammation, without lipid or blood pres-

Received March 19, 2021; accepted March 19, 2021; J-STAGE Advance Publication released online April 20, 2021

Division of Inflammation Research, Center for Molecular Medicine, Jichi Medical University, Shimotsuke, Japan

Mailing address: Masafumi Takahashi, MD, PhD, Division of Inflammation Research, Center for Molecular Medicine, Jichi

Medical University, 3311-1 Yakushiji, Shimotsuke 329-0498, Japan. E-mail: masafumi2@jichi.ac.jp

All rights are reserved to the Japanese Circulation Society. For permissions, please e-mail: cj@j-circ.or.jp

ISSN-1346-9843 
DAMPs \& PAMPs

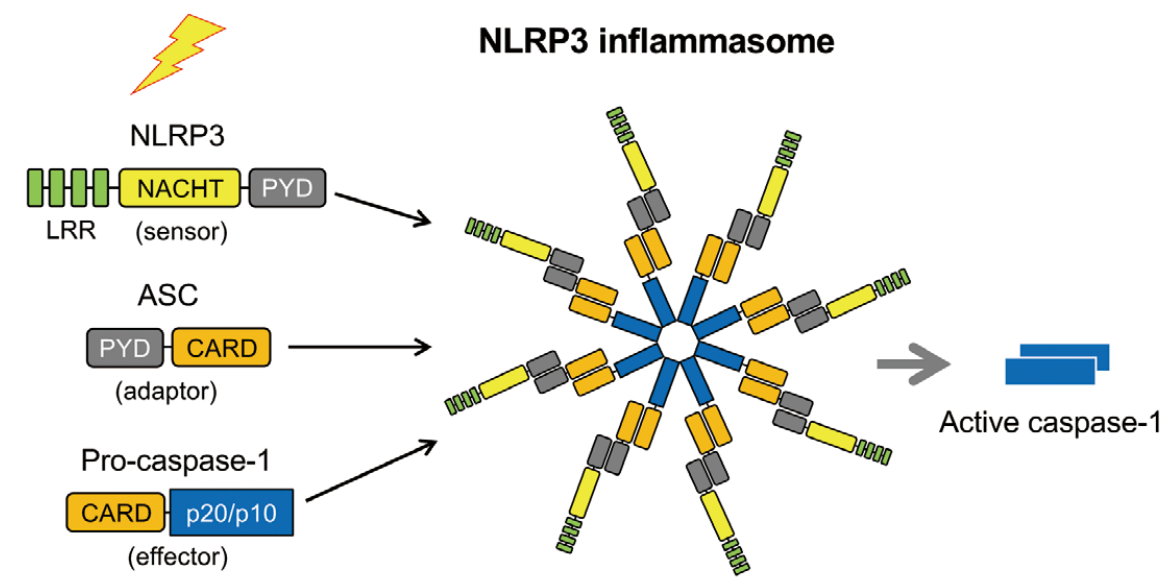

Figure 1. Nucleotide-binding oligomerization domain-like receptor family pyrin domain containing 3 (NLRP3) inflammasome assembly. The NLRP3 inflammasome consists of 3 components: the sensor NLRP3, the adaptor apoptosis-associated speck-like protein containing a caspase-recruitment domain (ASC), and the effector pro-caspase-1. When activated by damage/dangerassociated molecular patterns (DAMPs) or pathogen-associated molecular patterns (PAMPS), NLRP3 recruits and binds ASC through homotypic pyrin domain (PYD)-PYD interactions. In turn, ASC recruits and binds pro-caspase-1 through caspase-recruitment domain (CARD)-CARD interactions, leading to self-cleavage of pro-caspase-1 and release of its catalytically active subunits p20/10. LRR, leucine-rich repeat; NACHT, central nucleotide-binding and oligomerization domain.

sure lowering; ${ }^{13}$ this supports the concept that the NLRP3 inflammasome is a promising therapeutic target for CVD, including atherosclerosis and AAA.

This review summarizes the current knowledge, with a focus on the role of the NLRP3 inflammasome in atherosclerosis and AAA, and discusses the prospects of NLRP3 inflammasome-targeted therapy.

\section{Biology of the NLRP3 Inflammasome}

The term "inflammasome" was first described in 2002 as a cytosolic multiprotein signaling platform that activates inflammatory caspases. ${ }^{14}$ The inflammasome generally consists of 3 components: a pattern-recognition receptor (PRR) as a sensor, apoptosis-associated speck-like protein containing a caspase-recruitment domain (ASC) as an adaptor, and pro-caspase-1 as an effector (Figure 1). ${ }^{\mathbf{7 , 8 , 1 5}}$ To date, many inflammasome-forming PRRs have been reported, such as NLRP1, NLRP3, NLR family caspaserecruitment domain (CARD) containing 4 (NLRC4), absent in melanoma 2 (AIM2), and Pyrin. Each inflammasome is named after the sensor PRR and activated by different stimuli. The NLRP3 inflammasome is well characterized because it is formed in response to a broad range of stimuli, including PAMPs and DAMPs, and is implicated in the pathogenesis of sterile inflammatory diseases. When activated, NLRP3 recruits and binds ASC through homotypic pyrin domain (PYD)-PYD interactions, and ASC, in turn, recruits and binds pro-caspase-1 through CARD-CARD interactions, leading to self-cleavage and activation of pro-caspase-1 (Figure 1). In this process, ASC oligomerizes and forms a large "speck" $(\sim 1.0 \mu \mathrm{m}$ in diameter); therefore, the formation of an ASC speck is considered to be a hallmark of inflammasome activation.

Active caspase- 1 converts pro-IL- $1 \beta$ and pro-IL-18 to biologically active IL- $1 \beta$ and IL- 18 . Active caspase- 1 also converts gasdermin D (GSDMD) to GSDMD-N terminus (GSDMD-N), which, in turn, oligomerizes and forms pores on the plasma membrane. The GSDMD-forming pores not only lead to the release of cytosolic contents, including active IL- $1 \beta$ and IL-18, but also cause lytic cell death termed "pyroptosis". In addition to GSDMD, a recent study showed that caspase-3-mediated cleavage of GSDME (also known as DFNA5) can induce pyroptosis. ${ }^{\mathbf{1 6}}$ In this regard, we previously showed that NLRP3 inflammasome-driven pyroptosis occurs through an ASC/caspase-8/caspase-3 pathway under caspase-1 inhibition. ${ }^{17}$ Because this pyroptosis under caspase- 1 inhibition occurs in the absence of IL- $1 \beta$ release (a cardinal feature of pyroptosis), we call it incomplete pyroptosis. Pyroptosis has now been redefined as gasdermin-mediated lytic cell death. ${ }^{\mathbf{1 8}}$

Activation of the NLRP3 inflammasome requires 2 sequential signals: priming (signal 1) and activation (signal 2). ${ }^{7,8,15}$ Because protein levels of NLRP3 and pro-IL- $1 \beta$ are relatively low in resting cells, the priming signal transcriptionally upregulates these protein levels via Tolllike receptors (TLRs) or cytokine receptor-mediated activation of nuclear factor $(\mathrm{NF})-\kappa \mathrm{B}$. Post-translational modifications (PTMs), such as de-ubiquitination and phosphorylation, have also been shown to induce a rapid priming of the NLRP3 protein. Thereafter, the activation signal promotes assembly of the complex of the NLRP3 inflammasome and leads to caspase-1 activation. This 2-step signal is considered to be a fine-tuned regulation of NLRP3 inflammasome activation to avoid accidental release of the potent inflammatory mediator IL- $1 \beta$.

Previous studies have shown that NLRP3 is activated by a wide variety of stimuli, such as ATP, the bacterial poreforming toxin nigericin, and particulate matter or nanoparticles; therefore, researchers have assumed that NLRP3 


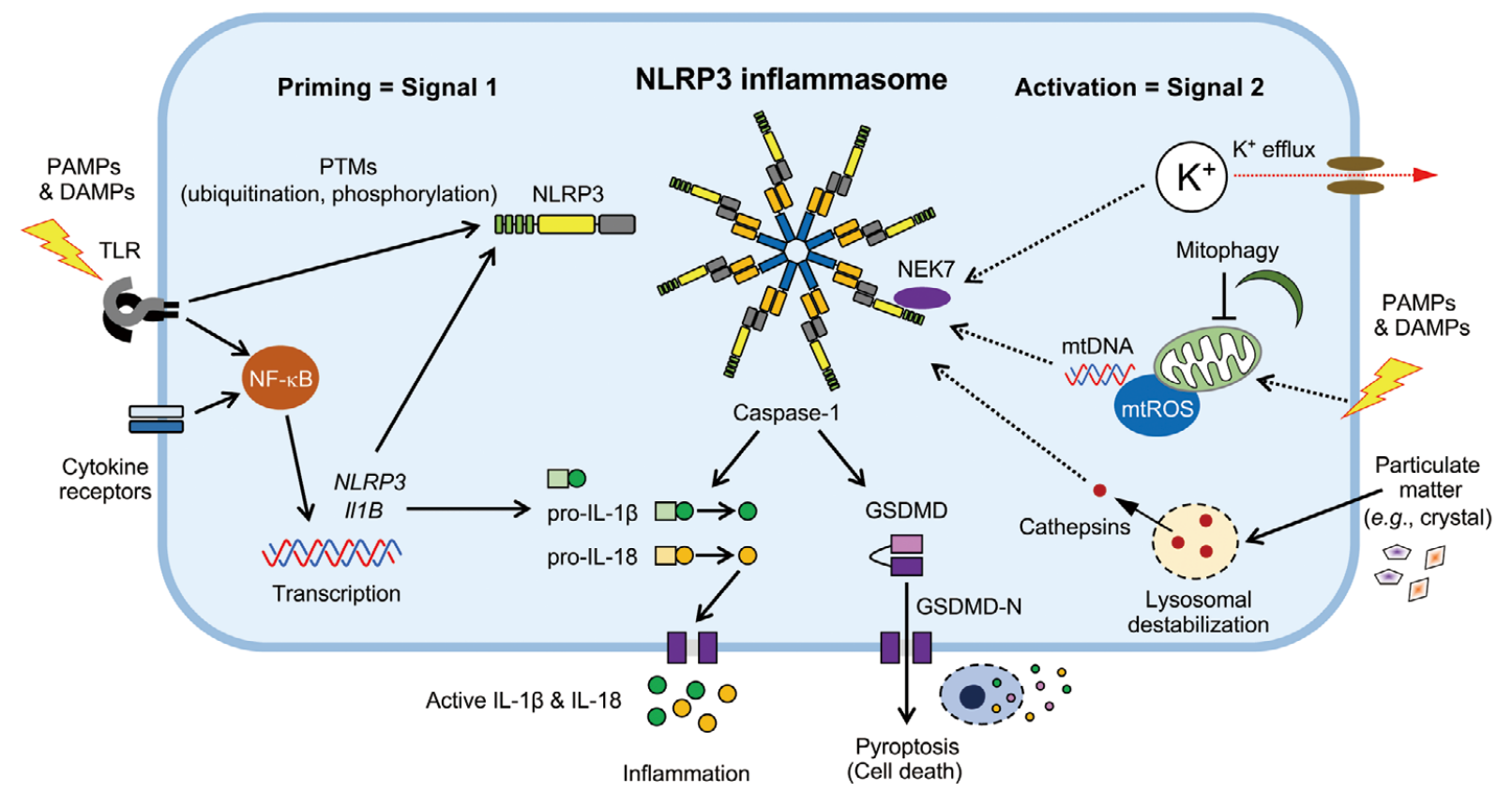

Figure 2. Mechanism of nucleotide-binding oligomerization domain-like receptor family pyrin domain containing 3 (NLRP3) inflammasome activation. NLRP3 inflammasome activation requires 2 sequential signals: priming (signal 1) and activation (signal 2). The priming signal induces the transcription of NLRP3 and pro-interleukin (IL)-1 $\beta$ via Toll-like receptors (TLRs) or cytokine receptormediated activation of nuclear factor (NF)-kB. Post-translational modifications (PTMs), such as de-ubiquitination and phosphorylation, have also been shown to induce rapid priming of the NLRP3 protein. Thereafter, the activation signal promotes assembly of the complex of the NLRP3 inflammasome and leads to caspase-1 activation. Several common upstream events for NLRP3 activation have been proposed, including $\mathrm{K}^{+}$efflux, cathepsin release by lysosomal damage, and mitochondrial reactive oxygen species (mtROS) and mitochondrial DNA (mtDNA). Mitophagy inhibits NLRP3 inflammasome activation by clearance of damaged mitochondria. NIMA-related kinase 7 (Nek7) binds NLRP3 and acts as an upstream activator of NLRP3 inflammasome assembly. Active caspase- 1 converts pro-IL-1 $\beta$ and pro-IL-18 to active IL-1 $\beta$ and IL-18, leading to inflammation. The active caspase- 1 also converts gasdermin D (GSDMD) to GSDMD-N terminus (GSDMD-N), which, in turn, oligomerizes and forms pores on the plasma membrane. The GSDMD-forming pores lead to the release of cytosolic contents (e.g., active IL-1 $\beta$ and IL-18) and cause lytic cell death (pyroptosis). DAMPs, damage/danger-associated molecular patterns; PAMPs, pathogen-associated molecular patterns.

acts as a global sensor of cellular stress and damage. From this point of view, several common events upstream of NLRP3 activation have been proposed, including intracellular potassium $\left(\mathrm{K}^{+}\right)$efflux, cathepsin release by lysosomal damage, and the production of mitochondrial reactive oxygen species and DNA (mtROS/mtDNA; Figure 2). Of these, $\mathrm{K}^{+}$efflux is considered to be a key common event to activate the NLRP3 inflammasome. ${ }^{19}$ Lysosomal damage followed by the release of cathepsins (e.g., cathepsin B) mainly contributes to NLRP3 inflammasome activation by particulate matter or nanoparticles. ${ }^{20}$ Some NLRP3-activating stimuli induce mitochondrial damage and subsequent production of $\mathrm{mtROS} / \mathrm{mtDNA}$, leading to NLRP3 inflammasome activation. ${ }^{21}$ Regarding reactive oxygen species (ROS), thioredoxin-interacting protein (TXNIP) has been reported to bind NLRP3 and induce its activation.22 In addition to mtROS, oxidized mtDNA, which is released from damaged mitochondria or is newly synthesized, causes NLRP3 inflammasome activation. ${ }^{23-25}$ Furthermore, these cellular events have been shown to be connected to each other; however, the precise mechanism by which NLRP3 can be activated remains elusive.

Recent studies have shown that the serine/threonine kinase NIMA-related kinase 7 (Nek7) directly binds
NLRP3 and acts as an upstream activator of NLRP3 inflammasome assembly. ${ }^{\mathbf{2 6 - 2 8}}$ Notably, Nek7-mediated NLRP3 activation is independent of Nek7 kinase activity. In addition, other ionic fluxes, including $\mathrm{Ca}^{2+}$ mobilization, $\mathrm{Na}^{+}$influx, and $\mathrm{Cl}^{-}$efflux, and phosphatidylinositol4-phosphate on a dispersed trans-Golgi network have been reported to contribute to NLRP3 activation. ${ }^{29}$ Furthermore, the priming and activation signals are fine-tuned by many regulators, including endogenous modulators (e.g., CARD-only proteins and pyrin-only proteins), PTMs (e.g., ubiquitination and phosphorylation), and microRNAs. ${ }^{30,31}$ Moreover, regulation of the NLRP3 inflammasome may vary in different cell types and with different stimuli. Readers are referred to recent reviews of the regulatory mechanism of the NLRP3 inflammasome., $7,8,15,32$

\section{NLRP3 Inflammasome and Atherosclerosis}

IL- $1 \beta$ is a key mediator of inflammation in atherosclerosis. IL-1 $\beta$ induces the expression of endothelial adhesion molecules, such as intercellular adhesion molecule-1 and vascular cell adhesion molecule-1, and promotes the adhesion and migration of monocytes/macrophages into the vascular wall. ${ }^{33}$ IL-1 $\beta$ also stimulates VSMCs to proliferate 

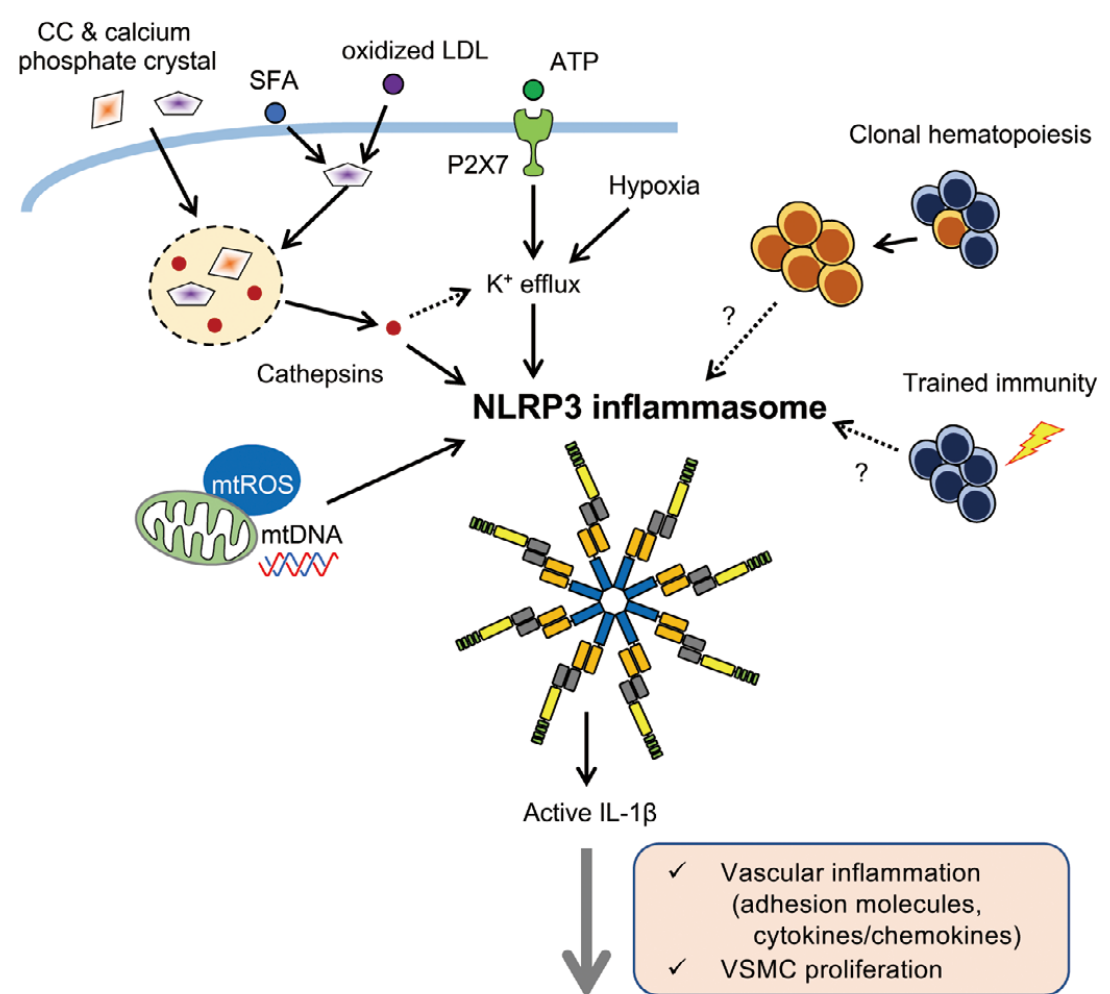

\section{Atherosclerosis}

Figure 3. Proposed mechanisms of nucleotide-binding oligomerization domain-like receptor family pyrin domain containing 3 (NLRP3) inflammasome activation in atherosclerosis. Cholesterol crystals (CCs) or calcium phosphate crystals are incorporated by the lysosome in macrophages. CCs or saturated fatty acid (SFA)-formed crystals are also formed intracellularly when oxidized low-density lipoprotein (LDL) or SFA is incorporated. These crystals cause lysosomal damage and cathepsin leakage, leading to NLRP3 inflammasome activation. NLRP3 inflammasome activation is also induced by ATP/P2X 7 - or hypoxia-mediated $\mathrm{K}^{+}$efflux and by the generation of mitochondrial reactive oxygen species (mtROS) and mitochondrial DNA (mtDNA). The release of active interleukin (IL)-1 $\beta$ following NLRP3 inflammasome activation induces vascular inflammation (e.g., upregulation of adhesion molecules and inflammatory cytokines/chemokines) and vascular smooth muscle cell (VSMC) proliferation, thus resulting in the development of atherosclerosis. The NLRP3 inflammasome may contribute to the association between atherosclerosis and trained immunity or clonal hematopoiesis.

and migrate into the intima and macrophages to produce other inflammatory cytokines and chemokines, such as IL-6 and C-C motif chemokine 2 (CCL2; also known as MCP-1), which further accelerates inflammation. ${ }^{34-36}$ Indeed, many animal studies have established the prominent contribution of IL- $1 \beta$ to atherogenesis. ${ }^{36-39}$ IL-18 has also been suggested to be a proatherogenic cytokine. ${ }^{40}$ Because the NLRP3 inflammasome is a main regulator of active IL- $1 \beta$ production, it had been presumed that it could be involved in the pathogenesis of atherosclerosis. Supporting this presumption, in 2008 we reported that the inflammasome adaptor ASC contributes to vascular inflammation and subsequent neointimal formation in a murine model of vascular injury; ${ }^{41}$ this indicates that the NLRP3 inflammasome may play a role in vascular inflammatory diseases, including atherosclerosis. The first study to investigate the NLRP3 inflammasome in atherosclerosis was reported by Latz's group in $2010 .{ }^{12}$ Using bone marrow-transplanted low-density lipoprotein receptor $(\mathrm{LDLR})^{-1-}$ mice, Duewell et al showed that bone marrow deficiency of NLRP3, ASC, or IL- $1 \alpha / \beta$ significantly reduced atherosclerotic lesion formation. ${ }^{\mathbf{1 2}}$ Along the same lines, it has been shown that systemic or bone marrow deficiency of caspase-1/11 decreases atherosclerotic lesions in apolipoprotein $\mathrm{E}(\mathrm{ApoE})^{-/-}$and $\mathrm{LDLR}^{-/-}$mice. $\mathbf{9 , 4 2}^{\mathbf{4}}$ Caspase-1/11 $1^{-/-}$mice have been widely used as caspase- $1^{-/-}$ mice, but they lack both caspase- 1 and caspase-11 due to the close proximity of the genomic loci. These reports indicate that NLRP3 inflammasome/IL- $1 \beta$ signaling plays a crucial role in the development of atherosclerosis. However, Menu et al reported that deficiency of NLRP3, ASC, and caspase-1/11 had no effect on atherosclerotic plaque size, macrophage infiltration, or plaque stability in $\mathrm{ApoE}^{-/-}$mice, ${ }^{43}$ which is in contrast with the studies mentioned above. Differences in the experimental conditions (e.g., atherogenic diet and sex) may underlie these conflicting results. In this regard, Chen et al recently suggested that NLRP3 inflammasome affects atherogenesis in LDLR $^{-1-}$ mice in a sex-specific manner. ${ }^{44}$ Studies using lentivirus-mediated NLRP3 gene silencing and the specific NLRP3 inhibitor MCC950 also showed that NLRP3 inflammasome has an atherogenic role. ${ }^{45,46}$ Furthermore, 


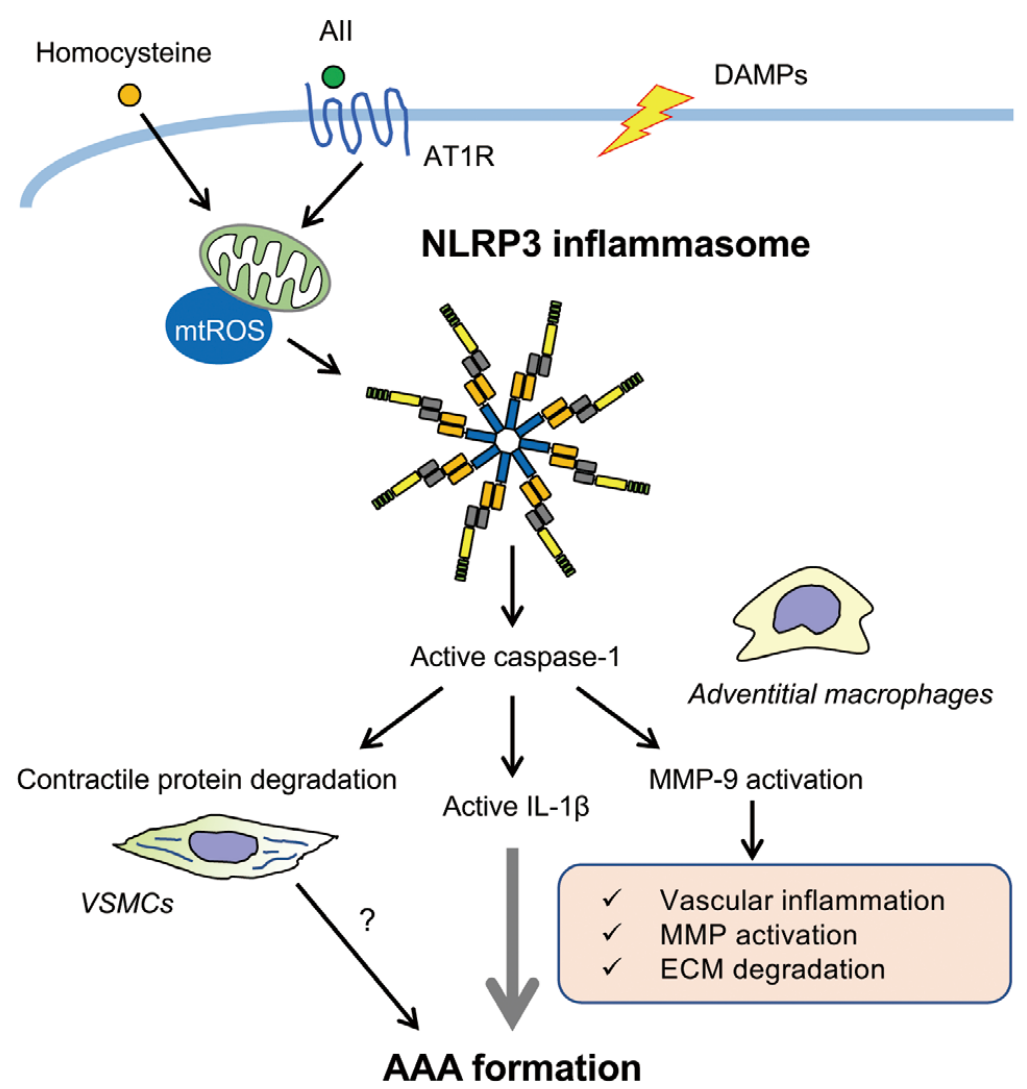

Figure 4. Proposed mechanisms of nucleotide-binding oligomerization domain-like receptor family pyrin domain containing 3 (NLRP3) inflammasome activation in abdominal aortic aneurysm (AAA). Based on our findings in an angiotensin (Ang) Il-infused AAA model, the following mechanism is proposed: Angll stimulates the generation of mitochondrial reactive oxygen species ( $m$ tROS) via the AT 1 receptor $\left(A T_{1} R\right)$, leading to NLRP3 inflammasome assembly and caspase-1 activation in adventitial macrophages. The release of active interleukin (IL)-1 $\beta$ causes vascular inflammation and the production of other inflammatory cytokines/ chemokines, further enhancing vascular inflammation. The enhanced inflammation induces matrix metalloproteinase (MMP) activation and extracellular matrix (ECM) degradation, resulting in the formation of AAA. Recent studies also suggest that active caspase-1 directly induces contractile protein degradation and MMP-9 activation in vascular smooth muscle cells (VSMCs), which can contribute to the development of AAA.

upregulation of NLRP3 inflammasome components has been shown in human atherosclerosis. ${ }^{47}$ Importantly, NLRP3 protein levels in peripheral leukocytes in acute coronary syndrome patients were correlated with the severity of coronary atherosclerosis. ${ }^{48}$

The molecular mechanisms and pathways of NLRP3 inflammasome activation in atherogenesis have been investigated extensively, including $\mathrm{K}^{+}$efflux, cathepsin release by lysosomal damage, and mtROS/mtDNA (Figure 3). Cholesterol crystals (CCs), which are frequently detected in the atherosclerotic plaques, are one of the most potent DAMPs to activate the NLRP3 inflammasome. ${ }^{\mathbf{1 2 , 2 0}} \mathrm{CCs}$ are phagocytosed by macrophages and then accumulated in lysosomes, which induces lysosomal damage and subsequent leakage of lysosomal enzyme cathepsins, resulting in NLRP3 inflammasome activation. CCs are also formed intracellularly by oxidized LDL, which is taken up into macrophages via CD36 scavenger receptor. In addition to CCs, calcium phosphate crystals or saturated fatty acid (SFA)-formed crystals also induce NLRP3 inflammasome activation.9,49 ATP is released extracellularly from dead or dying cells in the atherosclerotic necrotic core and strongly activates the NLRP3 inflammasome via the purinergic receptor $\mathrm{P} 2 \mathrm{X}_{7} / \mathrm{K}^{+}$efflux pathway. Studies using $\mathrm{P} 2 \mathrm{X}_{7}$-defi-

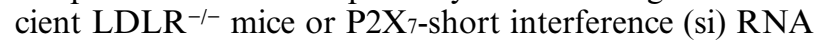
in $\mathrm{ApoE}^{-/-}$mice showed reductions in atherosclerotic lesions. ${ }^{50,51}$ Hypoxia is present in atherosclerotic plaques and has been reported to augment NLRP3 inflammasome activation in macrophages. ${ }^{\mathbf{2}}$ Regarding hypoxia, we have recently reported that NLRP3 inflammasome activation is promoted by $\mathrm{K}^{+}$efflux under hypoxia and glycose deprivation. ${ }^{53}$ Tumurkhuu et al reported that 8-oxoguanine glycosylase (OGG1), a major DNA glycosylase that eliminates oxidized DNA, has an atheroprotective role in $\mathrm{LDLR}^{-/-}$ mice. ${ }^{54}$ The expression of OGG1 was reduced in plaque macrophages, which increased cytosolic oxidized mtDNA, leading to NLRP3 inflammasome activation, suggesting that mtROS/mtDNA contribute to NLRP3 inflammasome activation in atherosclerosis. Many other stimuli have been reported to participate in the priming and activation signals of the NLRP3 inflammasome in the process of atherosclerosis. Interestingly, recent investigations have suggested 
that NLRP3 contributes to the association between atherosclerosis and trained immunity (innate immune memory) or clonal hematopoiesis. ${ }^{55-57}$ For detailed information, readers are referred to recent reviews. ${ }^{\mathbf{1 5 , 5 8 , 5 9}}$

\section{NLRP3 Inflammasome and AAA}

The mechanisms of AAA formation are complex and considered to include vascular inflammation, ROS production, extracellular matrix (ECM) degradation, thrombosis, and hemodynamic forces (Figure 4). ${ }^{5}$ IL-1 $\beta$ not only induces vascular inflammation, but also increases the activity of matrix metalloproteinases (MMPs), which degrade ECM components, including collagen and elastin. Furthermore, genetic deletion or pharmacological inhibition of IL- $1 \beta$ signaling decreases aortic aneurysm (AA) formation in several murine models. ${ }^{60-63}$ Regarding the NLRP3 inflammasome, a previous clinical study suggested that a genetic interaction between NLRP3 and CARD8 (a negative regulator of the NLRP3 inflammasome) confers a modest protective effect against AAA.$^{64} \mathrm{We}$ also found that ASC is highly expressed in macrophages infiltrating the adventitia of human AAA. ${ }^{30}$ Using a common AAA model of angiotensin (Ang) II-infused $\mathrm{ApoE}^{-/-}$mice, we showed that deficiency of NLRP3, ASC, and caspase-1/11 significantly reduced the incidence and severity of AAA, accompanied by inflammatory cytokine expression, adventitial macrophage infiltration, MMP-2/9 activation, and ECM degradation. We also showed that AngII activates the NLRP3 inflammasome and subsequently releases IL- $1 \beta$ via an AngII type 1 receptor (AT $\mathrm{R}$ )/mtROS-dependent pathway in macrophages. Consistent with these observations, Sun et al showed that lentivirus-mediated NLRP3 silencing decreased hyperhomocysteinemia-aggravated $\mathrm{CaCl}_{2}$-induced AAA formation in mice, and that the macrophage mtROS/NLRP3 inflammasome signaling mediates AAA formation in this model. ${ }^{65}$ Intriguingly, $\mathrm{Wu}$ et al reported that caspase-1 directly binds and cleaves contractile proteins, such as tropomyosin and myosin heavy chain, of VSMCs in vitro and that deficiency of NLRP3 and caspase-1 significantly reduced the degradation of these proteins and the formation of aneurysm and dissection in AngII-infused wild-type mice on a high-fat diet. ${ }^{66}$ More recently, the same research group showed that treatment with MCC950 prevented aortic dilatation, dissection, and rupture in different thoracic and abdominal aortic segments in AngII-infused wild-type mice fed a highfat and high-cholesterol diet. ${ }^{67}$ That study further showed that caspase-1 activated MMP-9 by direct cleavage of its $\mathrm{N}$-terminal inhibitory domain in macrophages. ${ }^{67}$ Conversely, studies using peripheral blood leukocytes of AAA patients showed that expression of inflammasome-related molecules was higher in leukocytes from male than female AAA patients, ${ }^{\mathbf{6}}$ suggesting that inflammasome activation in AAA may occur in a sex-dependent manner. Moreover, because AIM2 expression was obvious compared with NLRP3, it is suggested that the AIM2 inflammasome, rather than the NLRP3 inflammasome, contributes to the pathogenesis of human AAA ${ }^{\mathbf{6 8 , 6 9}}$ However, the role of the AIM2 inflammasome in AAA remains to be investigated.

\section{Clinical Trials Targeting Inflammation in CVD}

Recently, CANTOS showed that the anti-IL-1 $\beta$ antibody canakinumab significantly improved inflammatory status (i.e., serum levels of C-reactive protein [CRP] and IL-6) and reduced recurrent cardiovascular events in prior MI patients with residual inflammatory risk. ${ }^{13}$ The results of that trial suggest that IL- $1 \beta$-driven inflammation is a therapeutic target for atherosclerosis-related CVD. Two trials also targeting inflammation in CVD have been performed: the Cardiovascular Inflammation Reduction Trial (CIRT) and Colchicine Cardiovascular Outcome Trial (COLCOT). ${ }^{70,71}$ Although CIRT showed that low-dose methotrexate had no effects on serum concentrations of inflammatory markers (e.g., IL-1 $\beta$, IL-6, and CRP) or major cardiovascular events in prior MI patients with diabetes or metabolic syndrome, ${ }^{70}$ COLCOT showed a favorable outcome with the use of low-dose colchicine in patients with recent MI. ${ }^{71}$ Furthermore, the Low-Dose Colchicine 2 (LoDoCo2) trial very recently showed that low-dose colchicine reduced the risk of major cardiovascular events (i.e., primary MI and ischemia-driven revascularization) in patients with chronic coronary disease. ${ }^{72}$ Because colchicine inhibits assembly of the NLRP3 inflammasome by blocking microtubule polymerization, ${ }^{73}$ the results of COLCOT and the LoDoCo2 trial support the concept that the NLRP3 inflammasome is an upstream target for the prevention of atherosclerosis-related CVD. However, careful monitoring is needed to avoid adverse effects of long-term inhibition of the NLRP3 inflammasome/IL-1 $\beta$ pathway, such as infection and immune complications.

\section{Closing Remarks}

It is currently accepted that both atherosclerosis and AAA are chronic inflammatory diseases in the arterial wall. Although there are many mediators of inflammation, IL- $1 \beta$ is thought to be a key mediator for the development of atherosclerosis and AAA. The NLRP3 inflammasome has recently emerged as a key regulator of IL- $1 \beta$ production, and it has been shown that its inhibition attenuates the development of atherosclerosis and AAA. Furthermore, recent clinical trials, such as CANTOS, COLCOT, and LoDoCo2, have shown that targeting the NLRP3 inflammasome/IL-1 $\beta$ pathway may be effective for the prevention of atherosclerosis-related CVD. These observations suggest that the NLRP3 inflammasome is a common denominator of atherosclerosis and AAA. However, clinical evidence indicates that there are some differences between these disorders, suggesting that the mechanism of NLRP3 inflammasome activation differs. First, low-density lipoprotein is a major risk factor for atherosclerosis, but has no apparent association with AAA. Diabetes is also a risk factor for atherosclerosis, but is a negative or neutral risk factor for AAA ${ }^{4,5}$ Furthermore, although intimal atherosclerotic plaque and thrombosis are common features in both atherosclerosis and AAA, ECM degradation and adventitial chronic inflammation are predominantly observed in AAA rather than atherosclerosis. Therefore, we assume that different DAMPs may be engaged in atherosclerosis and AAA to activate the NLRP3 inflammasome. In this regard, the NLRP3 inflammasome in atherosclerosis has been vigorously studied for the past decade and its causative role is almost established. In particular, CCs are well-known DAMPs to activate the NLRP3 inflammasome. In contrast, the role of the NLRP3 inflammasome in the pathogenesis of AAA is not fully understood. One of the reasons for this is that murine models of AAA are limited and do not fully mimic human 
AAA. Although our previous study showed that AngII can act as a DAMP to activate the NLRP3 inflammasome in a murine AngII-infused AAA model, ${ }^{10}$ it is unclear whether AngII could play a similar role in the pathogenesis of human AAA. Second, atherosclerosis commonly affects large and medium-sized arteries, whereas AAA occurs in abdominal aortas., ${ }^{4,5}$ Because the NLRP3 inflammasome is expressed not only in innate immune cells, such as macrophages and neutrophils, but also in vascular cells, such as endothelial cells, VSMCs, and fibroblasts, it is possible that different cell types are responsible for activating the NLRP3 inflammasome in atherosclerosis and AAA. Thus, further investigations are needed to gain an understanding of the roles and mechanisms of the NLRP3 inflammasome in these disorders and to translate the experimental findings obtained in mice to humans.

\section{Acknowledgments}

The author thanks past and current members of the author's laboratory for their contributions to the research.

\section{Disclosures}

None.

\section{References}

1. Libby P, Okamoto Y, Rocha VZ, Folco E. Inflammation in atherosclerosis: Transition from theory to practice. Circ J 2010; 74: $213-220$.

2. Shimizu K, Mitchell RN, Libby P. Inflammation and cellular immune responses in abdominal aortic aneurysms. Arterioscler Thromb Vasc Biol 2006; 26: 987-994.

3. Daugherty A, Cassis LA. Mouse models of abdominal aortic aneurysms. Arterioscler Thromb Vasc Biol 2004; 24: 429-434.

4. Peshkova IO, Schaefer G, Koltsova EK. Atherosclerosis and aortic aneurysm: Is inflammation a common denominator? FEBS J 2016; 283: 1636-1652.

5. Golledge J, Norman PE. Atherosclerosis and abdominal aortic aneurysm: Cause, response, or common risk factors? Arterioscler Thromb Vasc Biol 2010; 30: 1075-1077.

6. Takahashi M. NLRP3 inflammasome as a novel player in myocardial infarction. Int Heart $J$ 2014; 55: 101-105.

7. Kelley N, Jeltema D, Duan YH, He Y. The NLRP3 inflammasome: An overview of mechanisms of activation and regulation. Int J Mol Sci 2019; 20: 3328.

8. Mangan MSJ, Olhava EJ, Roush WR, Seidel HM, Glick GD, Latz E. Targeting the NLRP3 inflammasome in inflammatory diseases. Nat Rev Drug Discov 2018; 17: 588-606.

9. Usui F, Shirasuna K, Kimura H, Tatsumi K, Kawashima A, Karasawa T, et al. Critical role of caspase-1 in vascular inflammation and development of atherosclerosis in Western diet-fed apolipoprotein E-deficient mice. Biochem Biophys Res Commun 2012; 425: 162-168.

10. Usui F, Shirasuna K, Kimura H, Tatsumi K, Kawashima A, Karasawa $\mathrm{T}$, et al. Inflammasome activation by mitochondrial oxidative stress in macrophages leads to the development of angiotensin II-induced aortic aneurysm. Arterioscler Thromb Vasc Biol 2015; 35: 127-136.

11. Karasawa T, Takahashi M. Role of NLRP3 inflammasomes in atherosclerosis. $J$ Atheroscler Thromb 2017; 24: 443-451.

12. Duewell P, Kono H, Rayner KJ, Sirois CM, Vladimer G, Bauernfeind FG, et al. NLRP3 inflammasomes are required for atherogenesis and activated by cholesterol crystals. Nature 2010; 464: $1357-1361$.

13. Ridker PM, Everett BM, Thuren T, MacFadyen JG, Chang WH, Ballantyne C, et al. Antiinflammatory therapy with canakinumab for atherosclerotic disease. $N$ Engl J Med 2017; 377: 1119-1131.

14. Martinon F, Burns K, Tschopp J. The inflammasome: A molecular platform triggering activation of inflammatory caspases and processing of prolL-beta. Mol Cell 2002; 10: 417-426.

15. Takahashi M. NLRP3 inflammasome as a key driver of vascular disease. Cardiovasc Res, doi:10.1093/cvr/cvab010.

16. Wang Y, Gao W, Shi X, Ding J, Liu W, He H, et al. Chemotherapy drugs induce pyroptosis through caspase- 3 cleavage of a gasder- min. Nature 2017; 547: 99-103.

17. Aizawa E, Karasawa T, Watanabe S, Komada T, Kimura H, Kamata R, et al. GSDME-dependent incomplete pyroptosis permits selective IL-1alpha release under caspase-1 inhibition. iScience 2020; 23: 101070.

18. Shi JJ, Gao WQ, Shao F. Pyroptosis: Gasdermin-mediated programmed necrotic cell death. Trends Biochem Sci 2017; 42: $245-254$.

19. Munoz-Planillo R, Kuffa P, Martinez-Colon G, Smith BL, Rajendiran TM, Nunez G. $\mathrm{K}^{+}$efflux is the common trigger of NLRP3 inflammasome activation by bacterial toxins and particulate matter. Immunity 2013; 38: 1142-1153.

20. Karasawa T, Takahashi M. The crystal-induced activation of NLRP3 inflammasomes in atherosclerosis. Inflamm Regen 2017; 37: 18 .

21. Zhou R, Yazdi AS, Menu P, Tschopp J. A role for mitochondria in NLRP3 inflammasome activation. Nature 2011; 469: 221-225.

22. Zhou RB, Tardivel A, Thorens B, Choi I, Tschopp J. Thioredoxininteracting protein links oxidative stress to inflammasome activation. Nat Immunol 2010; 11: 136-140.

23. Nakahira K, Haspel JA, Rathinam VA, Lee SJ, Dolinay T, Lam $\mathrm{HC}$, et al. Autophagy proteins regulate innate immune responses by inhibiting the release of mitochondrial DNA mediated by the NALP3 inflammasome. Nat Immunol 2011; 12: 222-230.

24. Zhong Z, Liang S, Sanchez-Lopez E, He F, Shalapour S, Lin XJ, et al. New mitochondrial DNA synthesis enables NLRP3 inflammasome activation. Nature 2018; 560: 198-203.

25. Shimada K, Crother TR, Karlin J, Dagvadorj J, Chiba N, Chen $\mathrm{S}$, et al. Oxidized mitochondrial DNA activates the NLRP3 inflammasome during apoptosis. Immunity 2012; 36: 401 -414.

26. He Y, Zeng MY, Yang DH, Metro B, Nunez G. NEK7 is an essential mediator of NLRP3 activation downstream of potassium efflux. Nature 2016; 530: 354-357.

27. Shi HX, Wang Y, Li XH, Zhan XM, Tang M, Fina M, et al. NLRP3 activation and mitosis are mutually exclusive events coordinated by NEK 7, a new inflammasome component. Nat Immunol 2016; 17: 250-258.

28. Schmid-Burgk JL, Chauhan D, Schmidt T, Ebert TS, Reinhardt J, Endl E, et al. A genome-wide CRISPR (clustered regularly interspaced short palindromic repeats) screen identifies NEK 7 as an essential component of NLRP3 inflammasome activation. $J$ Biol Chem 2016; 291: 103-109.

29. Chen JQ, Chen ZJJ. PtdIns4P on dispersed trans-Golgi network mediates NLRP3 inflammasome activation. Nature 2018; 564: $71-76$.

30. Karasawa T, Kawashima A, Usui F, Kimura H, Shirasuna K, Inoue Y, et al. Oligomerized CARD16 promotes caspase-1 assembly and IL-1beta processing. FEBS Open Bio 2015; 5: $348-356$.

31. Kawashima A, Karasawa T, Tago K, Kimura H, Kamata R, Usui-Kawanishi F, et al. ARIH2 ubiquitinates NLRP3 and negatively regulates NLRP 3 inflammasome activation in macrophages. J Immunol 2017; 199: 3614-3622.

32. Zheng D, Liwinski T, Elinav E. Inflammasome activation and regulation: Toward a better understanding of complex mechanisms. Cell Discov 2020; 6: 36.

33. Takahashi M, Ikeda U, Masuyama J, Kitagawa S, Kasahara T, Saito M, et al. Involvement of adhesion molecules in human monocyte adhesion to and transmigration through endothelial cells in vitro. Atherosclerosis 1994; 108: 73-81.

34. Takahashi M, Masuyama J, Ikeda U, Kasahara T, Kitagawa S, Takahashi Y, et al. Induction of monocyte chemoattractant protein-1 synthesis in human monocytes during transendothelial migration in vitro. Circ Res 1995; 76: 750-757.

35. Libby P, Warner SJ, Friedman GB. Interleukin 1: A mitogen for human vascular smooth muscle cells that induces the release of growth-inhibitory prostanoids. J Clin Invest 1988; 81: 487-498.

36. Libby P. Interleukin-1 beta as a target for atherosclerosis therapy: Biological basis of CANTOS and beyond. J Am Coll Cardiol 2017; 70: $2278-2289$.

37. Kirii H, Niwa T, Yamada Y, Wada H, Saito K, Iwakura Y, et al. Lack of interleukin-1beta decreases the severity of atherosclerosis in ApoE-deficient mice. Arterioscler Thromb Vasc Biol 2003; 23: 656-660.

38. Chi H, Messas E, Levine RA, Graves DT, Amar S. Interleukin-1 receptor signaling mediates atherosclerosis associated with bacterial exposure and/or a high-fat diet in a murine apolipoprotein $\mathrm{E}$ heterozygote model: Pharmacotherapeutic implications. Circulation 2004; 110: $1678-1685$.

39. Alexander MR, Moehle CW, Johnson JL, Yang Z, Lee JK, 
Jackson CL, et al. Genetic inactivation of IL-1 signaling enhances atherosclerotic plaque instability and reduces outward vessel remodeling in advanced atherosclerosis in mice. $J$ Clin Invest 2012; 122: 70-79.

40. Yasuda K, Nakanishi K, Tsutsui H. Interleukin-18 in health and disease. Int J Mol Sci 2019; 20: 649.

41. Yajima N, Takahashi M, Morimoto H, Shiba Y, Takahashi Y, Masumoto J, et al. Critical role of bone marrow apoptosisassociated speck-like protein, an inflammasome adaptor molecule, in neointimal formation after vascular injury in mice. Circulation 2008; 117: 3079-3087.

42. Gage J, Hasu M, Thabet M, Whitman SC. Caspase-1 deficiency decreases atherosclerosis in apolipoprotein E-null mice. Can J Cardiol 2012; 28: 222-229.

43. Menu P, Pellegrin M, Aubert JF, Bouzourene K, Tardivel A, Mazzolai L, et al. Atherosclerosis in ApoE-deficient mice progresses independently of the NLRP3 inflammasome. Cell Death Dis 2011; 2: e137.

44. Chen S, Markman JL, Shimada K, Crother TR, Lane M, Abolhesn A, et al. Sex-specific effects of the Nlrp3 inflammasome on atherogenesis in LDL receptor-deficient mice. JACC Basic Transl Sci 2020; 5: 582-598.

45. Zheng F, Xing S, Gong Z, Mu W, Xing Q. Silence of NLRP3 suppresses atherosclerosis and stabilizes plaques in apolipoprotein E-deficient mice. Mediators Inflamm 2014; 2014: 507208.

46. van der Heijden T, Kritikou E, Venema W, van Duijn J, van Santbrink PJ, Slutter B, et al. NLRP3 inflammasome inhibition by MCC 950 reduces atherosclerotic lesion development in apolipoprotein E-deficient mice: Brief report. Arterioscler Thromb Vasc Biol 2017; 37: 1457-1461.

47. Paramel Varghese G, Folkersen L, Strawbridge RJ, Halvorsen B, Yndestad A, Ranheim T, et al. NLRP3 inflammasome expression and activation in human atherosclerosis. J Am Heart Assoc 2016; 5: e003031.

48. Afrasyab A, Qu P, Zhao Y, Peng K, Wang H, Lou D, et al. Correlation of NLRP3 with severity and prognosis of coronary atherosclerosis in acute coronary syndrome patients. Heart Vessels 2016; 31: $1218-1229$.

49. Karasawa T, Kawashima A, Usui-Kawanishi F, Watanabe S, Kimura H, Kamata R, et al. Saturated fatty acids undergo intracellular crystallization and activate the NLRP3 inflammasome in macrophages. Arterioscler Thromb Vasc Biol 2018; 38: 744756.

50. Stachon P, Heidenreich A, Merz J, Hilgendorf I, Wolf D, Willecke $\mathrm{F}$, et al. $\mathrm{P}_{2} \mathrm{X}_{7}$ deficiency blocks lesional inflammasome activity and ameliorates atherosclerosis in mice. Circulation 2017; 135: $2524-2533$.

51. Peng K, Liu L, Wei D, Lv Y, Wang G, Xiong W, et al. P2 $\mathrm{X}_{7} \mathrm{R}$ is involved in the progression of atherosclerosis by promoting NLRP3 inflammasome activation. Int J Mol Med 2015; 35: $1179-1188$.

52. Folco EJ, Sukhova GK, Quillard T, Libby P. Moderate hypoxia potentiates interleukin-1 beta production in activated human macrophages. Circ Res 2014; 115: 875-883.

53. Watanabe S, Usui-Kawanishi F, Karasawa T, Kimura H, Kamata R, Komada T, et al. Glucose regulates hypoxia-induced NLRP3 inflammasome activation in macrophages. J Cell Physiol 2020; 235: 7554-7566.

54. Tumurkhuu G, Shimada K, Dagvadorj J, Crother TR, Zhang W, Luthringer D, et al. Ogg1-dependent DNA repair regulates NLRP3 inflammasome and prevents atherosclerosis. Circ Res 2016; 119: e76-e90.

55. Christ A, Gunther P, Lauterbach MAR, Duewell P, Biswas D, Pelka K, et al. Western diet triggers NLRP3-dependent innate immune reprogramming. Cell 2018; 172: 162-175.e114.

56. Fuster JJ, MacLauchlan S, Zuriaga MA, Polackal MN, Ostriker
AC, Chakraborty R, et al. Clonal hematopoiesis associated with TET2 deficiency accelerates atherosclerosis development in mice. Science 2017; 355: 842-847.

57. Sano S, Wang Y, Yura Y, Sano M, Oshima K, Yang Y, et al. JAK2 (V617F)-mediated clonal hematopoiesis accelerates pathological remodeling in murine heart failure. JACC Basic Transl Sci 2019; 4: 684-697.

58. Grebe A, Hoss F, Latz E. NLRP3 inflammasome and the IL-1 pathway in atherosclerosis. Circ Res 2018; 122: 1722-1740.

59. Stitham J, Rodriguez-Velez A, Zhang XY, Jeong SJ, Razani B. Inflammasomes: A preclinical assessment of targeting in atherosclerosis. Expert Opin Ther Targets 2020; 24: 825-844.

60. Johnston WF, Salmon M, Su G, Lu G, Stone ML, Zhao Y, et al. Genetic and pharmacologic disruption of interleukin-1beta signaling inhibits experimental aortic aneurysm formation. Arterioscler Thromb Vasc Biol 2013; 33: 294-304.

61. Johnston WF, Salmon M, Pope NH, Meher A, Su G, Stone ML, et al. Inhibition of interleukin-1 beta decreases aneurysm formation and progression in a novel model of thoracic aortic aneurysms. Circulation 2014; 130(Suppl 1): S51-S59.

62. Wakita D, Kurashima Y, Crother TR, Noval Rivas M, Lee Y, Chen $\mathrm{S}$, et al. Role of interleukin-1 signaling in a mouse model of Kawasaki disease-associated abdominal aortic aneurysm. Arterioscler Thromb Vasc Biol 2016; 36: 886-897.

63. Da Ros F, Carnevale R, Cifelli G, Bizzotto D, Casaburo M, Perrotta M, et al. Targeting interleukin-1 beta protects from aortic aneurysms induced by disrupted transforming growth factor beta signaling. Immunity 2017; 47: 959-973.e9.

64. Roberts RL, Van Rij AM, Phillips LV, Young S, McCormick SP, Merriman TR, et al. Interaction of the inflammasome genes CARD8 and NLRP3 in abdominal aortic aneurysms. Atherosclerosis 2011; 218: 123-126.

65. Sun W, Pang Y, Liu Z, Sun L, Liu B, Xu M, et al. Macrophage inflammasome mediates hyperhomocysteinemia-aggravated abdominal aortic aneurysm. J Mol Cell Cardiol 2015; 81: 96-106.

66. Wu D, Ren P, Zheng Y, Zhang L, Xu G, Xie W, et al. NLRP3 (nucleotide oligomerization domain-like receptor family, pyrin domain containing 3)-caspase-1 inflammasome degrades contractile proteins: Implications for aortic biomechanical dysfunction and aneurysm and dissection formation. Arterioscler Thromb Vasc Biol 2017; 37: 694-706.

67. Ren P, Wu D, Appel R, Zhang L, Zhang C, Luo W, et al. Targeting the NLRP3 inflammasome with inhibitor MCC950 prevents aortic aneurysms and dissections in mice. J Am Heart Assoc 2020; 9: e014044.

68. Wu X, Cakmak S, Wortmann M, Hakimi M, Zhang J, Bockler $\mathrm{D}$, et al. Sex- and disease-specific inflammasome signatures in circulating blood leukocytes of patients with abdominal aortic aneurysm. Mol Med 2016; 22: 505-518.

69. Wortmann M, Xiao X, Wabnitz G, Samstag Y, Hakimi M, Bockler D, et al. AIM2 levels and DNA-triggered inflammasome response are increased in peripheral leukocytes of patients with abdominal aortic aneurysm. Inflamm Res 2019; 68: 337-345.

70. Ridker PM, Everett BM, Pradhan A, MacFadyen JG, Solomon $\mathrm{DH}$, Zaharris E, et al. Low-dose methotrexate for the prevention of atherosclerotic events. N Engl J Med 2019; 380: 752-762.

71. Tardif JC, Kouz S, Waters DD, Bertrand OF, Diaz R, Maggioni AP, et al. Efficacy and safety of low-dose colchicine after myocardial infarction. $N$ Engl J Med 2019; 381: 2497-2505.

72. Nidorf SM, Fiolet ATL, Mosterd A, Eikelboom JW, Schut A, Opstal TSJ, et al. Colchicine in patients with chronic coronary disease. $N$ Engl J Med 2020; 383: 1838-1847.

73. Misawa T, Takahama M, Kozaki T, Lee H, Zou J, Saitoh T, et al. Microtubule-driven spatial arrangement of mitochondria promotes activation of the NLRP3 inflammasome. Nat Immunol 2013; 14: 454-460. 